\title{
Meso-Simulation and Experimental Research on the Mechanical Behavior of an Energetic Explosive
}

\author{
Qinxue Pan ${ }^{1, *(\mathbb{D})}$, Shuangyang $\mathrm{Li}^{1}$, Yang Liu ${ }^{2}$, Xiaoyu Xu ${ }^{1}$, Meile Chang ${ }^{1}$ and Yunmiao Zhang ${ }^{1}$ \\ 1 Key Laboratory of Fundamental Science for Advanced Machining, School of Mechanical Engineering, \\ Beijing Institute of Technology, Beijing 100081, China; 3120190343@bit.edu.cn (S.L.); \\ 3220180185@bit.edu.cn (X.X.); 15501267800@163.com (M.C.); dqzym97@163.com (Y.Z.) \\ 2 Metrology Physics and Chemistry Center, Inner Mongolia North Heavy Industry Group Co., Ltd., \\ Baotou 014030, China; janusliu@foxmail.com \\ * Correspondence: panqx@bit.edu.cn; Tel.: +86-010-68912714
}

Citation: Pan, Q.; Li, S.; Liu, Y.; Xu, X.; Chang, M.; Zhang, Y.

Meso-Simulation and Experimental Research on the Mechanical Behavior of an Energetic Explosive. Coatings 2021, 11, 64. https://doi.org/ 10.3390/coatings 11010064

Received: 14 December 2020

Accepted: 5 January 2021

Published: 7 January 2021

Publisher's Note: MDPI stays neutral with regard to jurisdictional clai$\mathrm{ms}$ in published maps and institutional affiliations.

Copyright: (C) 2021 by the authors. Licensee MDPI, Basel, Switzerland. This article is an open access article distributed under the terms and conditions of the Creative Commons Attribution (CC BY) license (https:// creativecommons.org/licenses/by/ $4.0 /)$.

\begin{abstract}
This study establishes a model for polymer-bonded explosives (PBX) using Digimat-FE. The model identifies the relationship between the material's effective elastic modulus and the explosive particle volume fraction, shape and gradation, and porosity, as well as other factors. Further, finite element analysis of the stress distribution of the PBX composite material is performed, and the mathematical models between the ultrasonic attenuation coefficient, particle volume fraction, and ultrasonic frequency are established. Finally, an efficient ultrasonic nondestructive testing system is designed to determine the stress distribution and fine crack groups in the material. Experimental results indicate that the relative error of stress detection is within $15 \%$, which meets the requirements of engineering applications.
\end{abstract}

Keywords: energetic explosive; effective elastic modulus; ultrasonic attenuation coefficient; residual stress; nondestructive testing

\section{Introduction}

Polymer-bonded explosives (PBXs) consist of a highly filled polymer matrix composite material. The production quality of PBXs affects the performance of the explosives; and its impact on weapon inventory, assembly, and storage is very complex. Consequently, $\mathrm{PBX}$ is the focus of considerable interest in the industrial production and scientific research fields. However, because of its complex mesoscopic composition, low strength, and energy-containing sensitivity, most studies on the meso-material properties of PBXs are in the theoretical and simulation stages. This mainly includes the viscoelasticity, mesodeformation damage, and effective elastic modulus. Residual stress is mainly responsible for the material defective damage. Residual stress is an elastic stress that is caused by an uneven plastic deformation inside the material that occurs in order to maintain its own balance. It is necessary to detect its value accurately; the ultrasonic method is considered to be one of the most promising technologies for this [1]. Liu et al. [2] used computer software and instrument hardware to develop a virtual instrument for ultrasonic testing; however, its residual stress detection accuracy could not meet the requirements for some materials. Zhou and Pei [3] developed a laser ultrasonic nondestructive testing platform to measure the stress of a PBX analog for material components; they obtained laser ultrasonic longitudinal wave signals with different loading stresses. However, owing to the large number of pores and inclusions in the composite material, the ultrasonic attenuation rate was high [4]. It was difficult to obtain a clear echo signal; hence, it was challenging to achieve nondestructive testing.

To carry out weapons safety research, we must fully understand the mechanical properties of PBX materials in different thermal environments [5]. Amongst others, the effective elastic modulus and ultrasonic attenuation coefficients are important factors. Dai 
and Liu [6] established a finite element model of the random distribution of round particles and the regular distribution of hexagonal particles based on the representative volume unit (RVE) method. In addition, they analyzed the effect of the shape for the explosive particles, along with the properties and volume fraction for the effective elastic modulus. Zhou et al. [7] established a two-dimensional RVE model consisting of explosive particles, a binder, and pores; they studied the effect of the pores on the effective elastic modulus of 2,4,6-Triamino-1,3,5-Trinitrobenzene-based (TATB) PBX. For the ultrasonic attenuation coefficient of PBX composite materials, Mu et al. [8] discussed the influence of porosity on the attenuation coefficient of casting polyurethane elastomer (CPUR). Zhou $[9,10]$ and Liu [11] assumed that the attenuation from each phase of the composite material is independent of other phases. From this assumption, they established a physical analysis model of the interaction between the various ultrasonic waves; however, they did not consider the model structure of the composite material. Instead, they only obtained the sound attenuation model through a simple superposition, which has major limitations.

PBX composite materials are characterized by their periodicity and symmetry [12]. Wang [13] and Xie [14] have analyzed and processed the mesoscopic structure of the composite materials by establishing an RVE model. By defining the constitutive model of single-phase materials and using the corresponding random algorithm, we can generate a random combination model of the material microstructure characteristic unit [15]. Uniform displacement boundary conditions can be applied and the RVE deformation, reaction force, and other information can be extracted to determine the RVE macro-material information. Afterward, finite element analysis can be performed using ABAQUS software (ABAQUS Inc., Providence, RI, USA).

In view of the low accuracy of residual stress detection and the limitations of the mechanical properties of PBX materials, in this study, Digimat-FE (Digimat 2017, MSC Software, Newport Beach, CA, USA) is used to establish a three-phase random distribution RVE model of PBX explosive particles, binder, and pores. This method is used to study the relationship between the material's effective elastic modulus and the explosive particle volume fraction, shape and gradation, and porosity to predict the effective elastic modulus. Then, ABAQUS is used to apply a stress load onto the pure binder and the RDX/Estane two-phase RVE model, respectively. Finite element analysis is performed to determine the stress distribution of the PBX composite, and mathematical models of the ultrasonic attenuation coefficient, particle volume fraction, and ultrasonic frequency are established. It is proven that scattering is the main factor of ultrasonic attenuation. Finally, an ultrasonic nondestructive testing system is designed to study the stress distribution and the fine crack groups in PBX composite materials with a high sampling frequency and to complete the evaluation of the material integrity. An ultrasonic experimental platform is also built to verify the established finite element model.

\section{Basic Theory}

\subsection{Ultrasonic Nondestructive Testing}

When ultrasonic waves pass through two media at different sound speeds, reflection, refraction, and waveform conversion will occur at the interface of the media. Ultrasonic waves can generate refracted longitudinal and refracted transverse waves in Medium 2, as shown in Figure 1 [16].

Among all the waves, the critically refracted longitudinal wave (LCR) propagating along the surface has the highest sensitivity to the material stress, the weakest attenuation, and the most noticeable waveform [17]. The incident angle of the ultrasonic wave is called the first critical angle $\theta_{c r}$. Therefore, it is most widely used during ultrasonic nondestructive testing.

The ultrasonic detection method for residual stress has been studied by applying the acoustoelastic theory. Some studies have determined that the relationship between the $\mathrm{L}_{\mathrm{CR}}$ wave velocity and stress propagating in the direction of the stress is represented by Equation (1) [18-20]: 


$$
\begin{aligned}
\rho v^{2}= & \lambda+2 \mu+\frac{\delta}{3 \lambda+2 \mu} \\
& {\left[\frac{\lambda+\mu}{\mu}(4 \lambda+10 \mu+4 m)+\lambda+21\right] }
\end{aligned}
$$

where $v$ is the longitudinal wave velocity whose propagation direction is consistent with the stress direction and $\rho$ is the density of the material. $\lambda$ and $\mu$ represents the second-order elastic constants of the material, whereas $l$ and $m$ represent the third-order elastic constants. $\sigma$ is the stress value; positive values indicate tensile stress, and negative values indicate compressive stress.

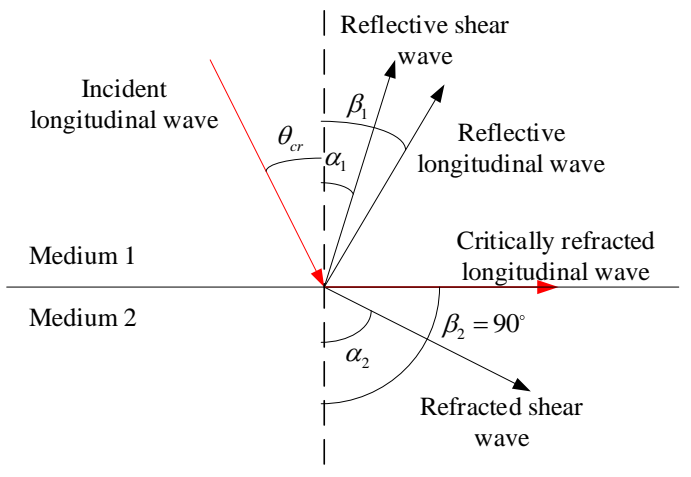

Figure 1. Wave conversion.

The relationship between the change in sound velocity and the change in stress is derived from Equation (2) [18-20]:

$$
\frac{v}{v_{0}^{2}} \frac{d v}{d \sigma}=\frac{k}{2}
$$

where $d \sigma$ represents the change in stress, $d v$ is the change in the propagation velocity of the $\mathrm{L}_{\mathrm{CR}}$ wave, $v_{0}$ is the propagation speed of the longitudinal wave under zero stress, and $k$ is the acoustic elastic constant.

When considering Equation (2), the relationship between the stress and sound velocity can be simplified [18-20]. By accurately measuring the LCR wave propagation time difference, the corresponding stress value can be calculated with Equation (3).

$$
d \sigma=K d t
$$

where $K=-2 / k t_{0}$ is the stress constant and $t_{0}=L / v_{0}$ is the time required for the $\mathrm{L}_{\mathrm{CR}}$ wave to propagate a fixed distance under zero stress.

\subsection{Acoustic Characteristics of the PBX Composite Materials}

The propagation characteristics of ultrasonic waves in a solid medium are determined by the elastic stiffness of the material; PBX includes explosive particles, binders, and other materials. The explosive particles and the binder will exhibit viscoelasticity under deformation, which represents the effective elastic modulus. Calculating the effective elastic modulus is very complicated and it depends on the ultrasonic frequency; therefore, it is necessary to establish an accurate model to predict the effective elastic modulus of the PBX material.

Studies have shown that the effective elastic modulus of PBX materials has an influence on the explosive particle volume fraction, shape and gradation, porosity, and other factors. The change in the effective elastic modulus with porosity can be expressed by Equation (4) [21]:

$$
E_{e f f}=a+b e^{-c \alpha},
$$

where $a, b$, and $c$ are three undetermined coefficients and $\alpha$ represents porosity.

Thus, this study establishes a three-phase RVE model of the PBX particles, binder, and pores based on Digimat-FE. This investigation also explores the effects of the explosive 
particle volume fraction, shape and gradation, and porosity on the effective elastic modulus of PBX materials.

Numerous experiments have demonstrated that the ultrasonic propagation characteristics of the RDX/Estane two-phase PBX are related to the viscoelasticity of the material, density, and frequency of the sound wave. As the density and frequency increase, the corresponding ultrasonic attenuation coefficient increases with a positive correlation [22,23]. In addition, the relationship between the attenuation coefficient and the ultrasonic frequency has a cubic polynomial fitting form:

$$
\alpha=a_{1} f^{3}+a_{2} f^{2}+a_{3} f+a_{4}
$$

where $f$ is the ultrasonic frequency and $\alpha$ is the ultrasonic attenuation coefficient.

However, the experimental results $[22,23]$ are only applicable to experimental systems and their associated equipment. In the modeling process for this study, RDX explosive particles and an Estane binder are used. Based on the linear elastic model and the viscoelastic constitutive model of the Prony series [24,25], the RDX/Estane two-phase polymer-bonded explosive model is used to simulate its acoustic properties.

\section{Results Predicting the Effective Elastic Modulus}

\subsection{Three-Phase RVE Model of the PBX Particles, Binder, and Pores}

The average diameter of the PBX particles is approximately $100 \mu \mathrm{m}$, so the RVE size was also chosen as $100 \mu \mathrm{m}$. Using the random distribution program in Digimat, a microscopic model of the explosive particle volume fraction that gradually changes is established (as shown in Figure 2). The explosive particles are represented by random circles, including two graded explosive particles with diameters of 80 and $150 \mu \mathrm{m}$. The blue and green circles are explosive particles, the light purple circles are pores, and the remaining white area represents the binder.

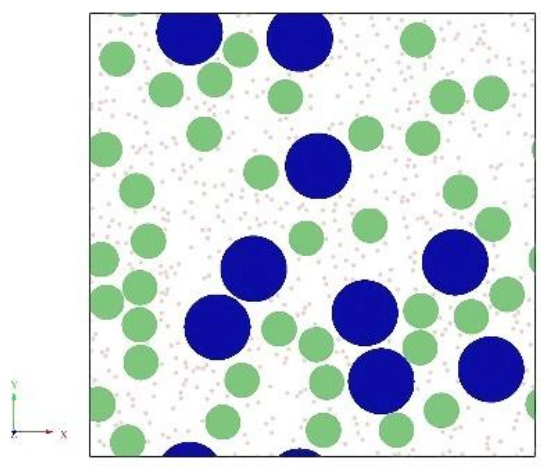

Figure 2. Schematic diagram of the microstructure of polymer-bonded explosives (PBX) explosive material.

Only the effective elastic modulus of the PBX was determined; the explosive particles and the binder are considered to be isotropic linear elastic materials. The explosive particles mainly include TATB, cyclotrimethylenetrinitramine (RDX), and cyclotetramethylenetetranitramine (HMX), whereas the binders include Estane, F2311, and F2314. Their material parameters are listed in Table 1.

Table 1. Material parameters of the explosive particles and binder.

\begin{tabular}{cccccc}
\hline & \multicolumn{2}{c}{ Explosive Particles } & \multicolumn{2}{c}{ Binder } \\
Material & Poisson's Ratio & Elastic Modulus (MPa) & Material & Poisson's Ratio & Elastic Modulus (MPa) \\
\hline TATB & 0.25 & 8860 & Estane & 0.495 & 13.2 \\
RDX & 0.21 & 18,400 & F2311 & 0.499 & 42.2 \\
HMX & 0.32 & 26,410 & F2314 & 0.33 & 397 \\
\hline
\end{tabular}




\subsection{Calculating the PBX Effective Elastic Modulus}

We applied periodic boundary conditions to the RVE model. Coupling constraints are applied to the right and upper boundaries. The movement of the left boundary in the $x$-direction is restricted, whereas the movement in the $y$-direction is free. The movement of the lower boundary in the $y$-direction is restricted, and the movement in the $x$-direction is free; thus, the shape of the RVE section remains unchanged after compression and deformation. In addition, based on the plane strain assumption, in order to simulate a uniaxial compression test, a compression displacement load with a strain of 0.1 is applied to the upper boundary. The boundary conditions and displacement load are shown in Figure 3.

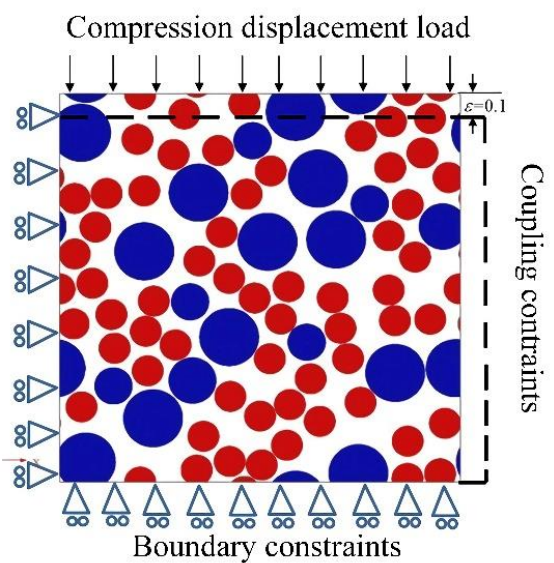

Figure 3. Boundary conditions and load application.

According to the plane strain hypothesis theory, the average stress and average strain of the RVE model in various directions can be obtained from the average value of the volume integral, as shown in Equations (6) and (7).

$$
\begin{aligned}
& \bar{\sigma}_{i}=\int_{V} \sigma_{i} d V / V \\
& \bar{\varepsilon}_{i}=\int_{V} \varepsilon_{i} d V / V,
\end{aligned}
$$

where $i$ represents the direction of the stress and strain and $V$ represents the volume of the RVE model. In the two-dimensional model, the relationship between the strain and stress is given by Equations (8) and (9).

$$
\begin{aligned}
\varepsilon_{x} & =\left(\sigma_{x}-v \sigma_{y}\right) / E_{e f f} \\
\varepsilon_{y} & =\left(\sigma_{y}-v \sigma_{x}\right) / E_{e f f},
\end{aligned}
$$

where $\sigma_{x}$ and $\sigma_{y}$ represent the stresses in the $x$ - and $y$-directions, respectively, and $\varepsilon_{x}$ and $\varepsilon_{y}$ represent the strains in the $x$ - and $y$-directions, respectively, $v$ represents the material's Poisson's ratio, and $E_{\text {eff }}$ represents the effective elastic modulus.

In Equations (6)-(9), the effective elastic modulus can be calculated using Equation (10).

$$
E_{e f f}=\left(\bar{\sigma}_{y}^{2}-\bar{\sigma}_{x}^{2}\right) /\left(\bar{\sigma}_{y} \bar{\varepsilon}_{y}-\bar{\sigma}_{x} \bar{\varepsilon}_{x}\right) .
$$

\subsection{Factors Influencing the Effective Elastic Modulus}

\subsubsection{Volume Fraction of the Explosive Particles}

This study establishes a two-phase PBX model that contains only random round explosive particles and a binder. In addition, the finite element analysis of PBX, consisting of nine different explosive particles and the binder, is carried out. The average stress and 
strain values in the $x$ - and $y$-directions are derived from the calculation results, and the effective elastic modulus is calculated according to Equation (10). Table 2 lists the effective elastic modulus of PBX with nine types of explosive particles and binder combinations. Figure 4 shows the curve of the effective elastic modulus of PBX versus the volume fraction of the explosive particles.

Table 2. Effective elastic modulus of the polymer-bonded explosives (PBX) with different explosive particles and binder.

\begin{tabular}{|c|c|c|c|c|c|c|c|c|}
\hline \multirow{2}{*}{ Explosive Composition } & \multicolumn{8}{|c|}{$E_{\text {eff }} \mathbf{M P a}$} \\
\hline & $30 \%$ & $40 \%$ & $50 \%$ & $60 \%$ & $65 \%$ & $70 \%$ & $75 \%$ & $80 \%$ \\
\hline TATB+Estane & 217.69 & 242.51 & 426.67 & 534.26 & 832.68 & 1145.67 & 1507.34 & 1823.1 \\
\hline TATB+F2311 & 410.48 & 464.67 & 664.74 & 1232.57 & 1666.5 & 2033.41 & 2451.34 & 2834.7 \\
\hline TATB+F2314 & 1365.31 & 1957.78 & 2617.17 & 3694.53 & 4292.14 & 4856.67 & 5454.35 & 6034.7 \\
\hline RDX+Estane & 485.23 & 591.98 & 712.99 & 815.19 & 1144.81 & 1465.45 & 1900.54 & 2253.3 \\
\hline $\mathrm{RDX}+\mathrm{F} 2311$ & 509.77 & 708.67 & 997.72 & 1696.36 & 2056.25 & 2399.78 & 2740.24 & 3133.5 \\
\hline $\mathrm{RDX}+\mathrm{F} 2314$ & 1793.12 & 2726.42 & 3706.86 & 5423.14 & 7236.41 & 8766.53 & $10,273.5$ & 11,832 \\
\hline HMX+Estane & 318.41 & 676.07 & 832.04 & 1014.72 & 1470.98 & 1830.79 & 2256.24 & 2676.1 \\
\hline $\mathrm{HMX}+\mathrm{F} 2311$ & 639.38 & 1026.83 & 1207.76 & 1742.64 & 2920.11 & 3450.34 & 3876.4 & 4232.7 \\
\hline $\mathrm{HMX}+\mathrm{F} 2314$ & 2548.39 & 3011.07 & 3846.85 & 7123.67 & 7629.4 & 9201.12 & $11,377.5$ & 12,565 \\
\hline
\end{tabular}

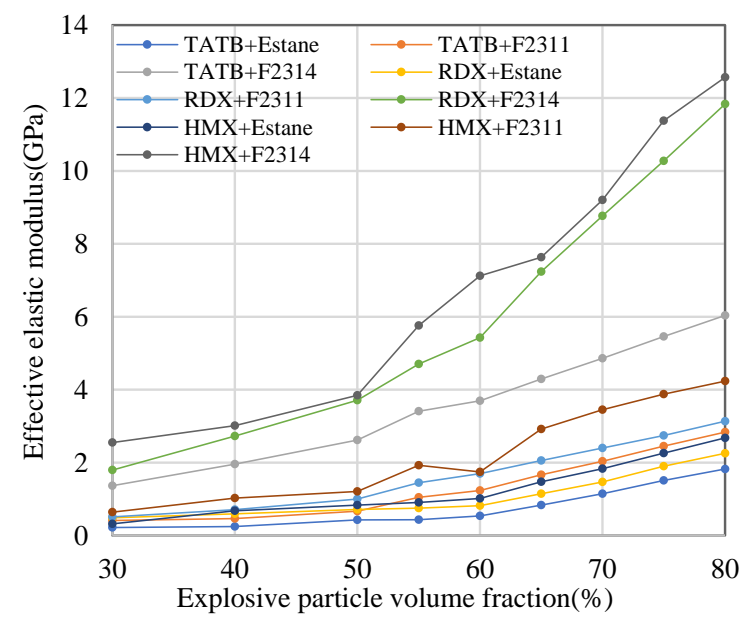

Figure 4. Influential curve of the volume fraction on the effective elastic modulus of PBX.

It can be observed that the effective elastic modulus increases as the volume fraction of the PBX particles increases. When the volume fraction is relatively small (less than 60\%), the increase rate is relatively small because the main part of the load and the deformer is the binder. Conversely, when the volume fraction is greater than $60 \%$, more explosive particles are subjected to load and deformation, and the rate of increase is significantly elevated. In addition, when the volume fractions of the explosive particles are the same, the effective elastic modulus of the PBX increases with the elastic modulus of the explosive particles and the binder.

\subsubsection{Shape and Gradation of the Explosive Particles}

The RVE model of PBX with different explosive particle shapes and gradations is established, as shown in Figure 5 [5]. The particle size of the two-level RVE model is 150 and $80 \mu \mathrm{m}$, and the particle size of the three-level RVE model is 200, 150, and $100 \mu \mathrm{m}$. The volume fraction of the explosive particles is $50 \%$.

Table 3 lists the effective elastic modulus of PBX for the different particle shapes and gradations when the particle volume fraction ranges from 30\% to 60\%. In this case, the explosive components are HMX and Estane. It can be observed that the gradation has a certain effect on the effective elastic modulus of the PBX. The effective elastic modulus of the PBX increases with the gradation of the explosive particles. It can be inferred that there 
is an optimal gradation that maximizes the mechanical properties of the PBX, whereas the shape of the explosive particles has little effect.

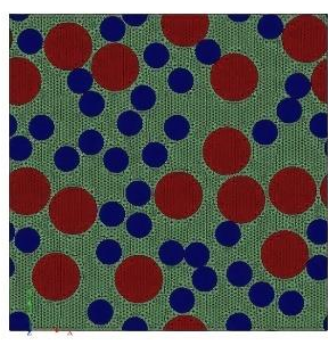

(a)

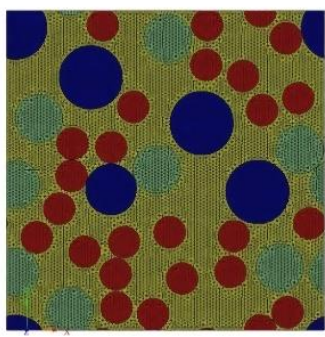

(b)

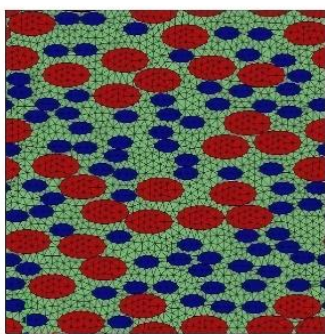

(c)

Figure 5. Representative volume unit (RVE) finite element model with different particle shapes and gradations. (a) Two grades and round particles, (b) three grades and round particles, and (c) two grades and oval particles.

Table 3. Effective elastic modulus of different shapes and gradations.

\begin{tabular}{cccc}
\hline \multirow{2}{*}{ Volume Fraction } & \multicolumn{3}{c}{ Effective Elastic Modulus (MPa) } \\
& 2 Grades & 3 Grades & $\begin{array}{c}\text { Oval Particle } \\
\text { 2 Grades }\end{array}$ \\
\hline $30 \%$ & 318.41 & 389.45 & 309.22 \\
$40 \%$ & 676.04 & 756.02 & 654.74 \\
$50 \%$ & 832.04 & 911.55 & 821.91 \\
$60 \%$ & 1014.72 & 1217.83 & 1012.21 \\
\hline
\end{tabular}

\subsubsection{Porosity}

In this study, the particles are RDX and the binder is Estane; the particle sizes are 80 and $150 \mu \mathrm{m}$, respectively; the volume fraction is $40 \%$, and the pore diameter of the binder is $0.01 \mathrm{~mm}$. The effective elastic modulus of the PBX corresponding to the RDX particles with different porosities was determined. By combining Equation (4), the fitting curve between the effective elastic modulus and the porosity for PBX is illustrated in Figure 6. Among them, $R^{2}$ represents the correlation coefficient between the approximating functions and measurement points. It can be observed that the existence of pores causes the effective elastic modulus to decrease significantly; there is an exponential relationship with the porosity.

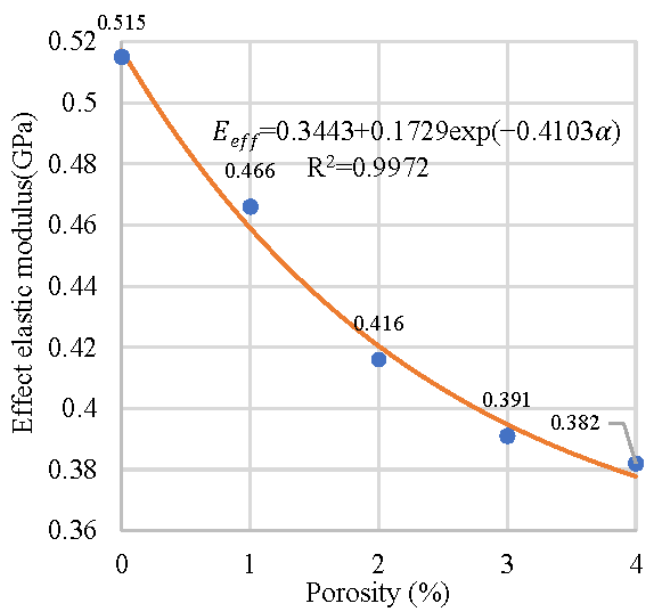

Figure 6. Effect of porosity on the effective elastic modulus of cyclotrimethylenetrinitramine (RDX)based PBX explosives. 


\section{Simulation Calculation of the Ultrasonic Attenuation Coefficient 4.1. RDX/Estane Two-Phase RVE Model}

In this model, the minimum sound wave frequency was set to $1 \mathrm{MHz}$, and the RVE unit size was determined to be $2 \times 2 \mathrm{~mm}^{2}$. Using the random distribution available in Digimat-FE, three types of mesoscopic models with explosive particle volume fractions of $10 \%, 40 \%$, and $60 \%$ are established. Figure 7 shows a PBX model with an explosive particle volume fraction of $40 \%$, in which the round particles are explosive particles with diameters of 80 and $150 \mu \mathrm{m}$, and the remaining white area represents the binder.

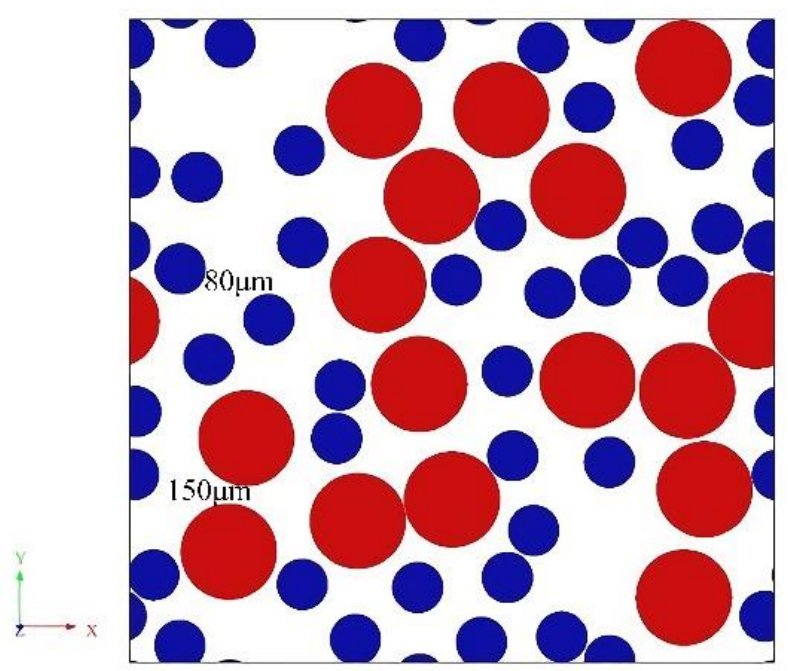

Figure 7. Random round particle distribution geometric model with volume fraction of $40 \%$.

Based on the representative units, they are gridded in ABAQUS through related interfaces. The size of the grid determines the accuracy of the numerical calculations. On the premise of ensuring that the accuracy meets the requirements, in order to reduce the complexity of meshing, we choose to uniformly use a grid dimension that is for $80-\mu \mathrm{m}$ particles. In general, the grid size must satisfy the following condition:

$$
d \leq v /(8 f),
$$

where $d$ is the maximum size of the grid, $v$ is the ultrasonic velocity inside the material, and $f$ is the ultrasonic frequency. In this model, the theoretical grid size should not exceed $26 \mu \mathrm{m}$. The chosen $20-\mu \mathrm{m}$ grid is a four-node bilinear plane strain quadrilateral element (CPE4R), which reduces the integral.

\subsection{Ultrasonic Loading and Attenuation Coefficient Calculation Principle}

According to the sound attenuation coefficient measuring method in engineering [12], the constraint is applied in the form of a supported beam, and ultrasonic excitation is applied on the upper surface as loading, as shown in Figure 8. We chose ultrasound with center frequencies of $1,2.5,4,5,6$, and $8 \mathrm{MHz}$ to study the ultrasound attenuation of each frequency.

In addition, the Hanning window function was used to modulate the single-frequency sinusoidal signal. In terms of wave energy, the sine wave modulated by the Hanning window has low attenuation characteristics. When this modulated wave is used for ultrasonic testing, the additional attenuation can be minimized. At this time, the most accurate ultrasonic attenuation coefficient can be obtained by obtaining the effective peak value of the ultrasonic wave entering the material.

The ultrasonic attenuation coefficient is obtained by recording the previous and current pressure envelope wave peak values, $B_{0}$ and $B_{1}$. This can be determined by applying Equation (12) [12]. 


$$
\alpha=(20 / h) \times \ln \left(B_{0} / B_{1}\right),
$$

where $h$ is the distance between the two collection points.

We imported the model into ABAQUS/EXPLICIT for signal extraction and processing and obtained the sound attenuation coefficients at different frequencies and particle volume fractions.

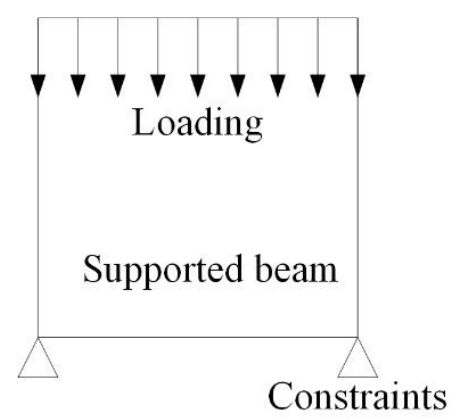

Figure 8. Model constraints and loading.

\subsection{Finite Element Analysis of the Sound Field for the RVE Model}

\subsubsection{Pure Binder RVE Model}

After meshing the model and adding loads and constraints, a $1 \mathrm{MHz}$ stress wave is applied to obtain a cloud image of the sound pressure. As shown in Figure 9, the sound wave is a five-peak sine wave that is modulated by a Hanning window. The ultrasonic attenuation rate inside the material is calculated by obtaining the effective peak value of the ultrasonic wave after entering the material.
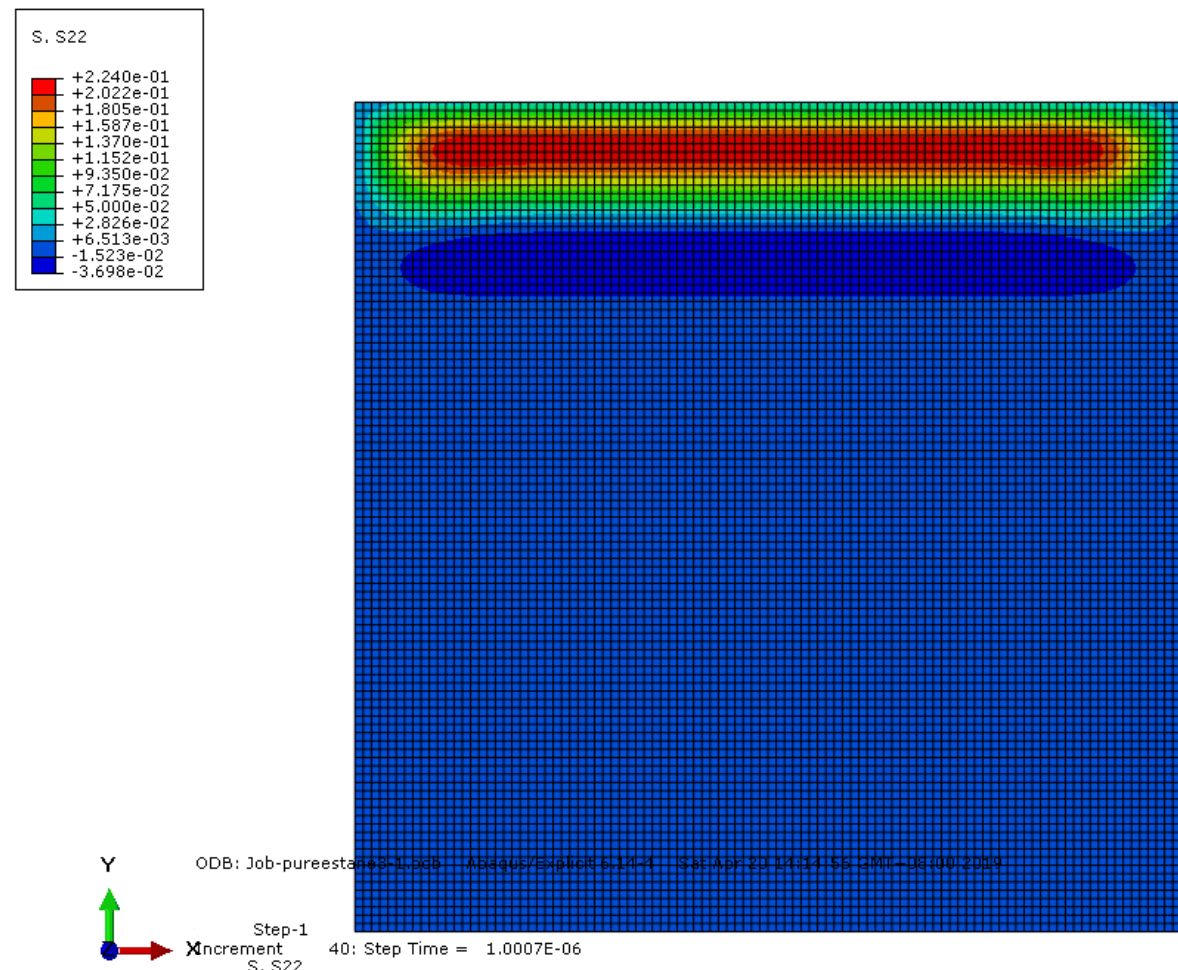

(a)

Figure 9. Cont. 


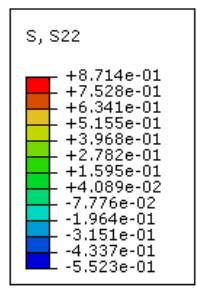

(b)

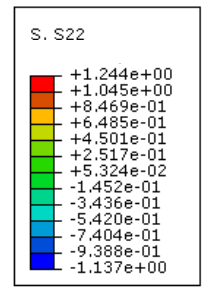

$\underset{\substack{\text { Step-1 } \\ \text { S, S22 }}}{\substack{\text { ODB: Job-pure } \\ \text { Stent }}}$

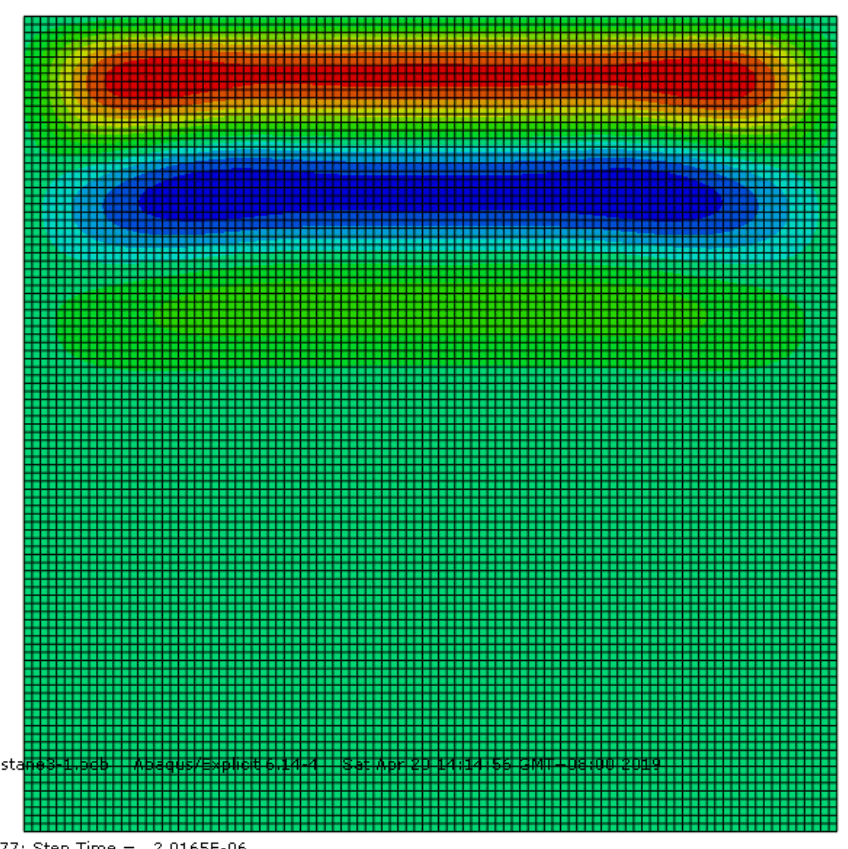

7: Step Time $=2.0165 \mathrm{E}-06$

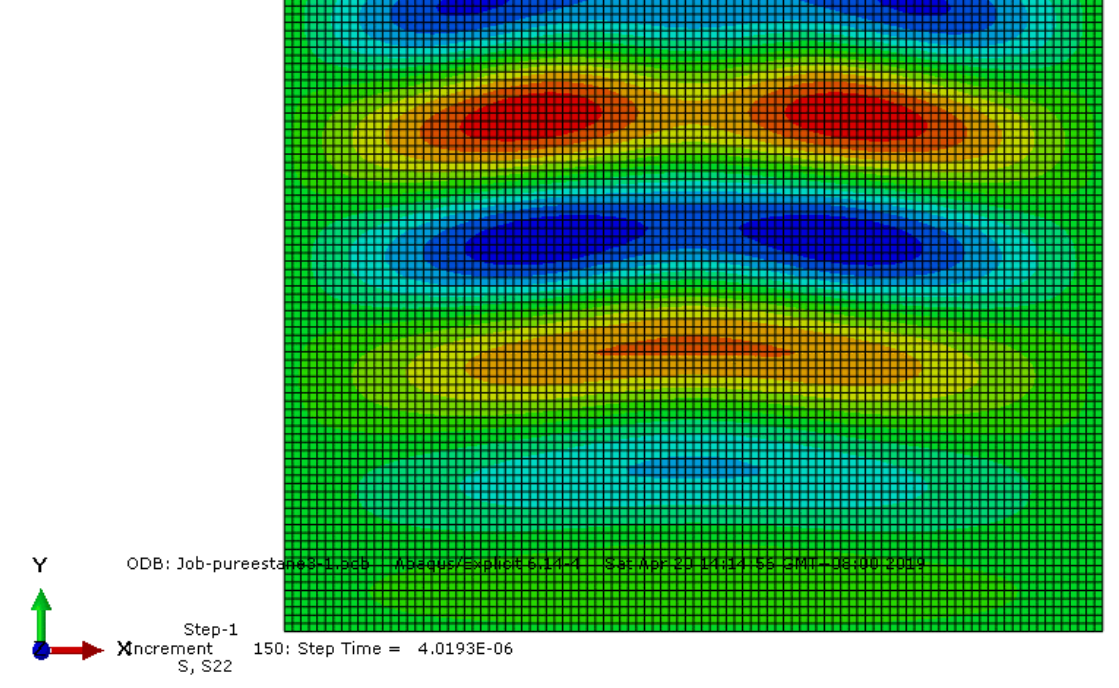

(c)

Figure 9. Cont. 


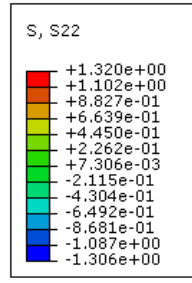

\section{,}

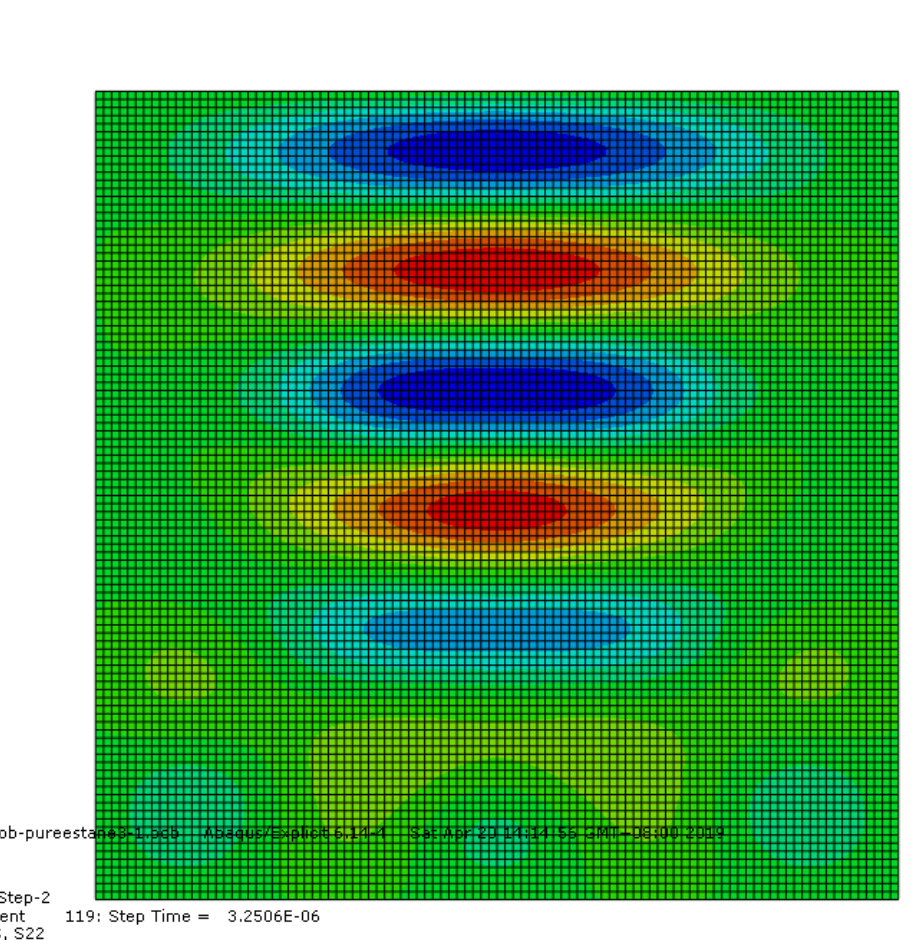

(d)

Figure 9. Cloud propagation of a $1 \mathrm{MHz}$ ultrasonic wave in the binder (Estane) after (a) 1, (b) 2, (c) 4, and (d) $8.25 \mu \mathrm{s}$.

In addition, a finite element simulation of the ultrasonic attenuation of the pure binder was established and compared with the later two-phase RDX/Estane material to determine the main factors affecting the ultrasonic attenuation coefficient. From the finite element analysis, we obtained the attenuation coefficients at different frequencies using Equation (12). The attenuation coefficient is roughly proportional to the ultrasonic frequency, as shown in Figure 10. Identically, $R^{2}$ represents the correlation coefficient between the approximating functions and measurement points.

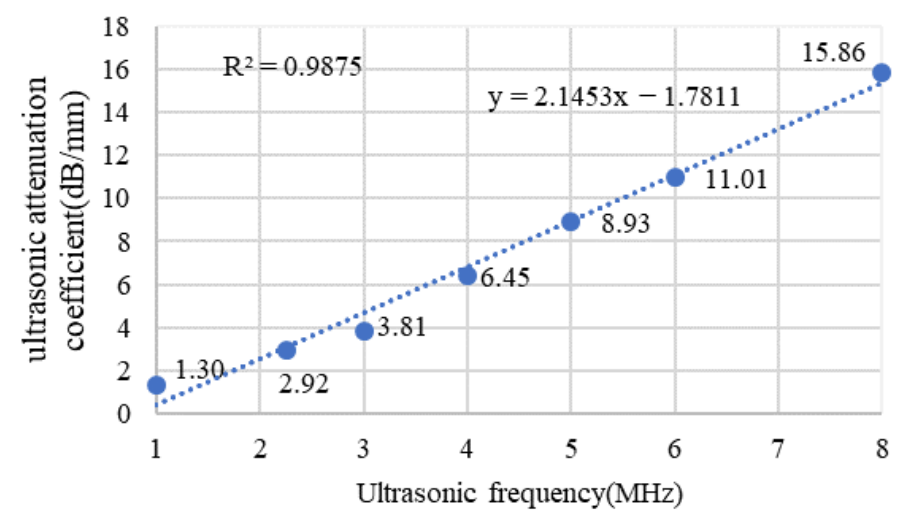

Figure 10. Relationship between the ultrasonic attenuation coefficient and the frequency of the Estane binder.

\subsubsection{Single-Particle RVE Model of the Different RDX Particle Volume Fractions}

When ultrasonic waves propagate in composite viscoelastic materials, the acoustic properties of the medium also have a very important influence on the amplitude of the ultrasonic signals. The attenuation mechanism of the sound waves is more complicated. 
These mechanisms comprehensively affect the attenuation of the ultrasonic waves, but the effects are independent of each other. They are formed by the linear superposition of the viscous attenuation, heat conduction attenuation, and scattering attenuation.

$$
\alpha=\beta_{\eta} \alpha_{\eta}+\beta_{\xi} \alpha_{\xi}+\beta_{s} \alpha_{s},
$$

where $\alpha_{\eta}, \alpha_{\xi}$, and $\alpha_{s}$ represent the viscous, heat conduction, and scattering attenuation coefficients, respectively. $\beta_{\eta}, \beta_{\tilde{\xi}}$, and $\beta_{s}$ represent their respective influential factors on the total attenuation coefficient.

The influencing factor of the scattering attenuation coefficient is much greater than the other two attenuation coefficients. Therefore, in this RDX/Estane study, the focus is on the impact of ultrasonic scattering. Based on the previous RVE model construction process, the RVE model of the explosive particles with a particle size of $100 \mu \mathrm{m}$ is established. A $1 \mathrm{MHz}$ stress wave was applied to the model, and the sound pressure distribution cloud diagram was obtained, as shown in Figure 11.

According to Equation (12), the ultrasonic attenuation coefficients of the different frequencies in the single-particle model can be obtained and compared with the pure Estane binder. The results are shown in Table 4. It can be concluded that when the ultrasonic frequency is small, the sound attenuation coefficients of the two RVE models have little difference. From a macroscopic perspective, the coefficient is determined by the modulus and the density of the material, and the existence of a single particle has little effect on these two properties. From a mesoscopic perspective, when the radius of the explosive particle is approximately equal to the wavelength $\lambda$, the equation to determine the attenuation coefficient of the surface sound intensity scattering is expressed as follows:

$$
\Delta \alpha_{s}=\frac{\pi}{16} k_{1}^{2} R_{s}^{2} g_{s} n_{0},
$$

where $n_{0}$ is the number of explosive particles per unit volume, $R_{s}$ is the particle radius, $k_{1}$ is the wave number, and $g_{s}$ is the attenuation constant. For this single-particle model, $R_{s}=$ $50 \mu \mathrm{m}, n_{0}=1$, and $\Delta \alpha_{s} \approx 0$, which has almost no effect on the attenuation coefficient.

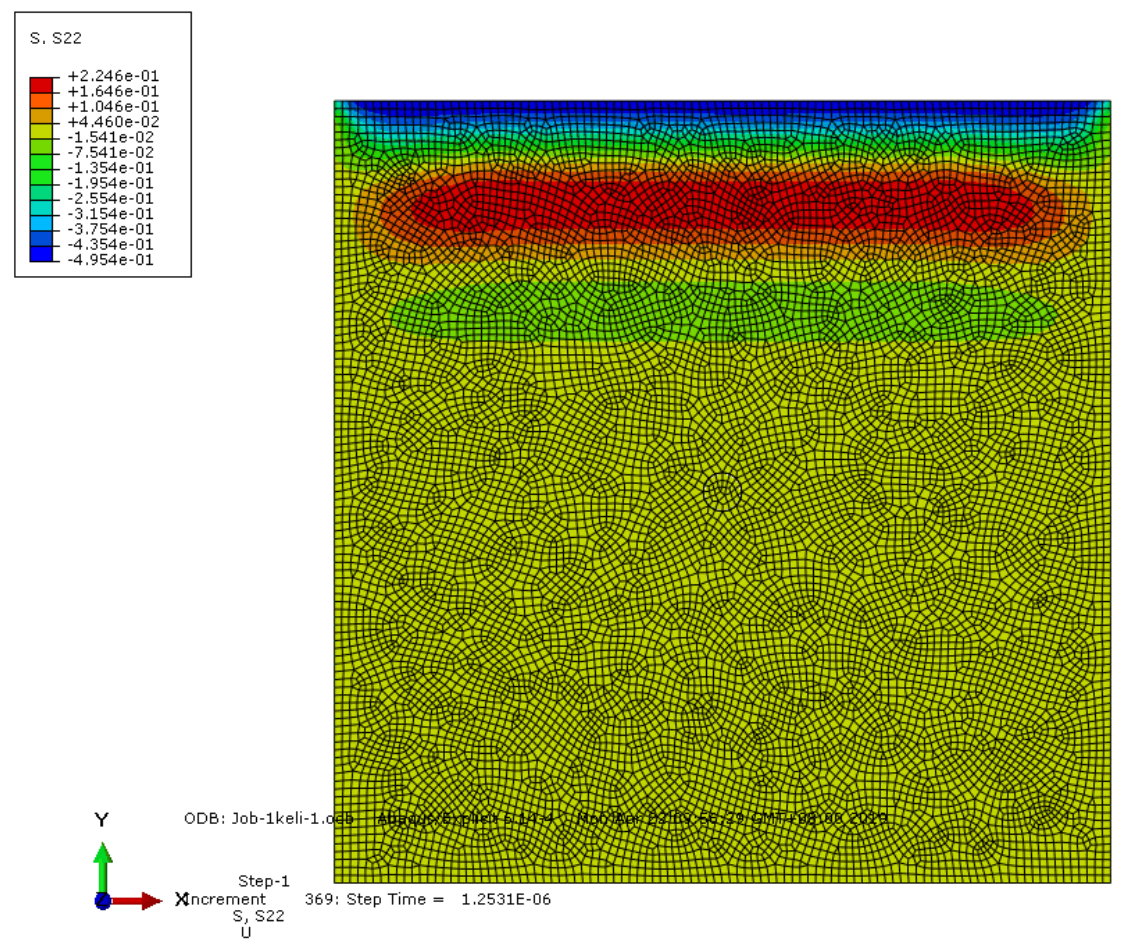




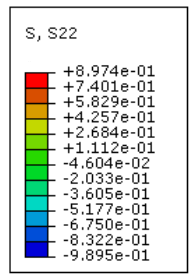

(b)

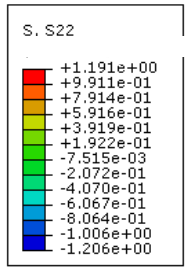

Step-1
S

660: Step Time $=2.2534 \mathrm{E}-06$

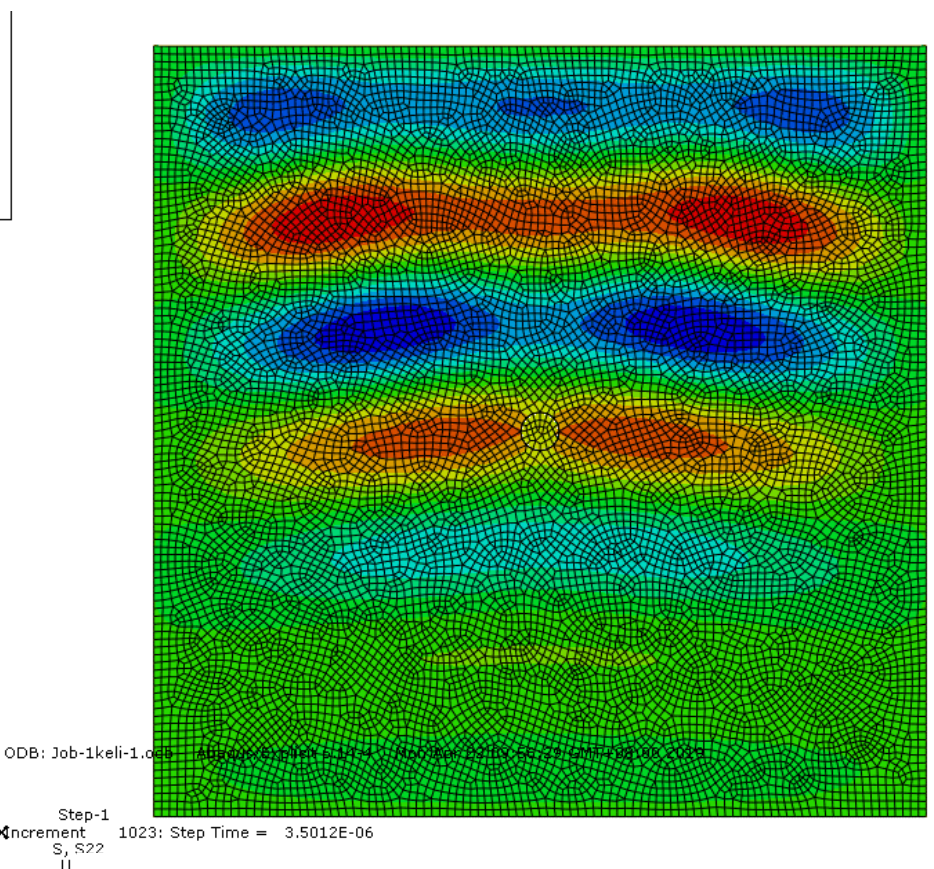

(c)

Figure 11. Cont. 


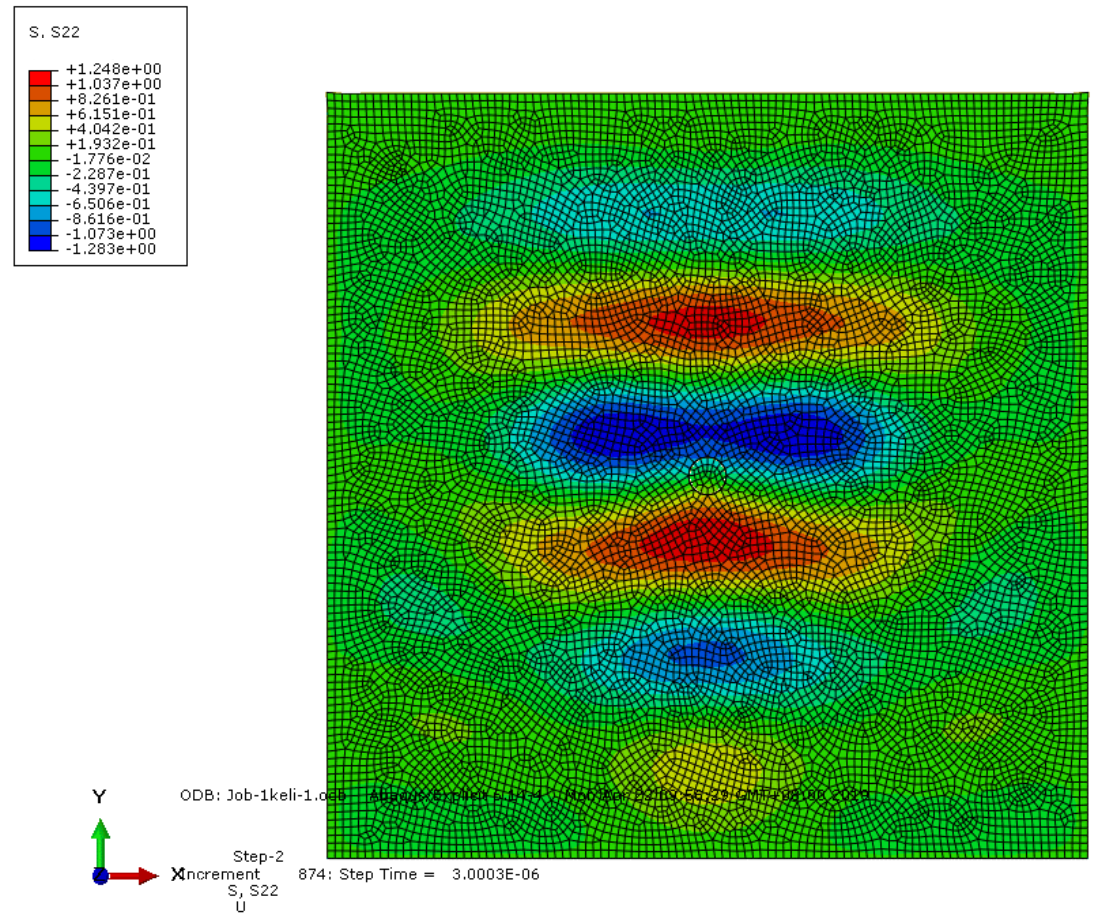

(d)

Figure 11. Cloud diagram of the $1 \mathrm{MHz}$ ultrasonic propagation process in a single-particle RVE model after (a) 1.2531, (b) 2.2534 , (c) 3.5012, and (d) $8.0003 \mu \mathrm{s}$.

Table 4. Ultrasonic attenuation coefficients of the two representative volume unit (RVE) models.

\begin{tabular}{ccccc}
\hline RVE Model & Ultrasonic Frequency $\mathbf{( M H z )}$ & Single Particle & Estane Binder & Difference \\
\hline & 1 & 1.402 & 1.302 & 0.1 \\
& 2.25 & 2.354 & 2.326 & 0.028 \\
Sound attenuation coefficient & 3 & 4.440 & 3.866 & 0.574 \\
$(\mathrm{~dB} / \mathrm{mm})$ & 4 & 8.348 & 6.445 & 1.903 \\
& 5 & 12.482 & 9.145 & 3.337 \\
& 6 & 16.809 & 1.013 & 5.796 \\
\hline
\end{tabular}

Conversely, as the frequency increases, the sound attenuation coefficient of the singleparticle RVE model is significantly greater than the Estane binder RVE model. This is because the wavelength of the ultrasonic waves gradually becomes smaller than the size of the explosive particles, the reflection and absorption are more obvious, and the energy attenuation is greater.

The RVE model with RDX particle volume fractions of $10 \%, 40 \%$, and $60 \%$ was analyzed in the sound field, and the sound attenuation coefficient was calculated. The results obtained are shown in Figure 12.

It can be observed that in the same RVE model, the ultrasonic attenuation coefficient increases with ultrasonic frequency, and there is a nonlinear relationship between them. When the RVE model is loaded with ultrasonic waves of the same frequency, the ultrasonic attenuation coefficient becomes larger with the volume fraction of explosive particles. The results obtained by the finite element method are consistent with the subsequent experimental results, which proves the rationality of the finite element model. 


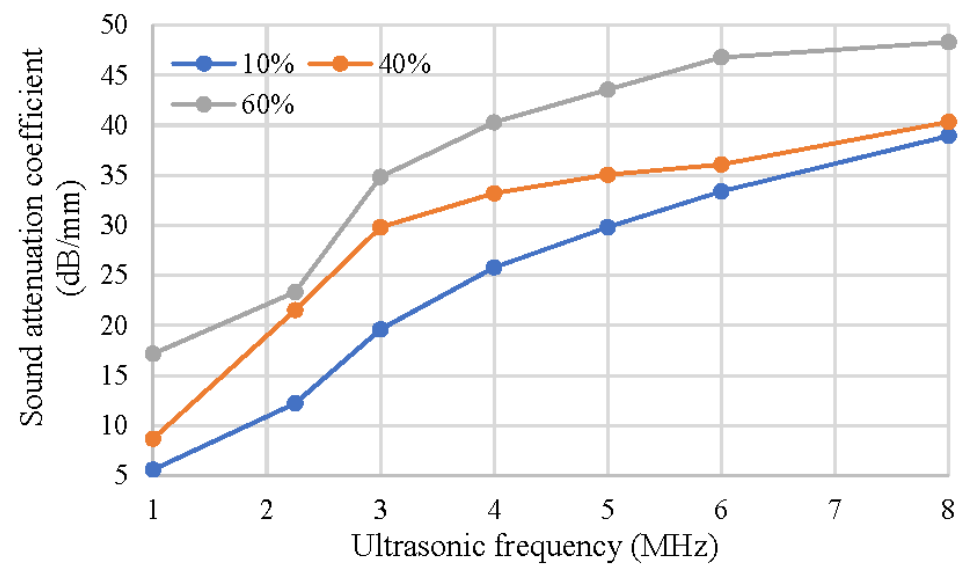

Figure 12. Ultrasonic attenuation coefficient of the different particle volume fractions with an ultrasonic frequency.

\section{System Design}

The ultrasonic nondestructive system mainly consists of ultrasonic testing cards, ultrasonic transducers, industrial computers, and additional software modules, as shown in Figure 13.

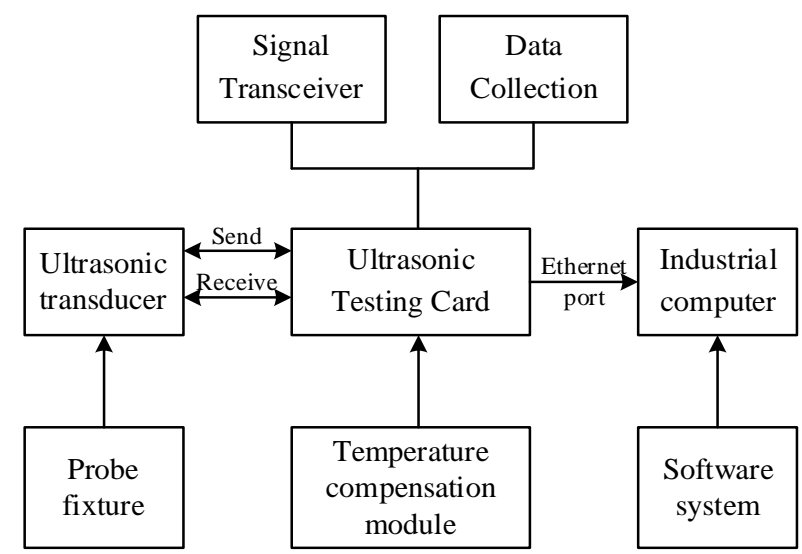

Figure 13. Detection system.

\subsection{Transducers}

\subsubsection{Ultrasound Probe}

In terms of a direct signal transmission and a receiving device for composite materials, an ultrasonic probe with an in-band impedance transformer, a preamplifier, and an adjustable inductance is required to ensure a constant basic natural frequency and to avoid interference from other harmonic components.

\subsubsection{Wedge}

To produce an LCR wave, the sound velocity of the incident medium must be lower than that of the refracting medium. In our experiment, the internal sound velocity of the PBX composite material is at least $2800 \mathrm{~m} / \mathrm{s}$. Therefore, we chose polytetrafluoroethylene (PTFE), with a sound velocity of $1422 \mathrm{~m} / \mathrm{s}$ as the wedge material. According to Snell's theorem, the first critical angle can be determined using Equation (15):

$$
\theta_{c r}=\sin ^{-1}\left(v_{1} / v_{2}\right),
$$

where $v_{1}$ refers to the propagation speed of the obliquely incident longitudinal wave in the first medium, $v_{2}$ refers to the propagation speed of the refracted longitudinal wave in the second medium, and $\theta_{c r}$ refers to the first critical angle. 
The first critical angle that is suitable for this system is $30.5^{\circ}$. Previous studies have shown that when the incident angle is $1^{\circ}$ greater than the first critical angle, the displacement amplitude of the $\mathrm{L}_{\mathrm{CR}}$ wave is largest [26]. Therefore, the incident wedge angle after the correction is $32^{\circ}$, as shown in Figure 14.

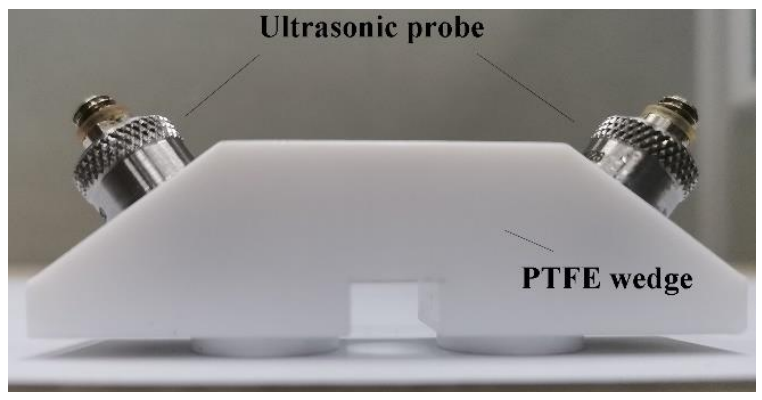

Figure 14. Polytetrafluoroethylene (PTFE) wedge and an ultrasonic probe.

\subsection{Signal Transceiver}

This system uses the ZXUS-80SM ultrasonic pulse transceiver card produced by Zhongxu Technology Co., Ltd., Wuhan, China, which integrates the pulse transmitter, signal receiver and data acquisition end. Besides, the data acquisition card realizes the A/D conversion of ultrasonic analog signal and computer digital signal.

\subsection{Inspection System Software Add-on Module}

The ultrasonic detection system software was developed using $\mathrm{C}++$. The software uses a graphic language, which mainly include a parameter setting module, a data processing module, and a detection algorithm module, as shown in Figure 15.

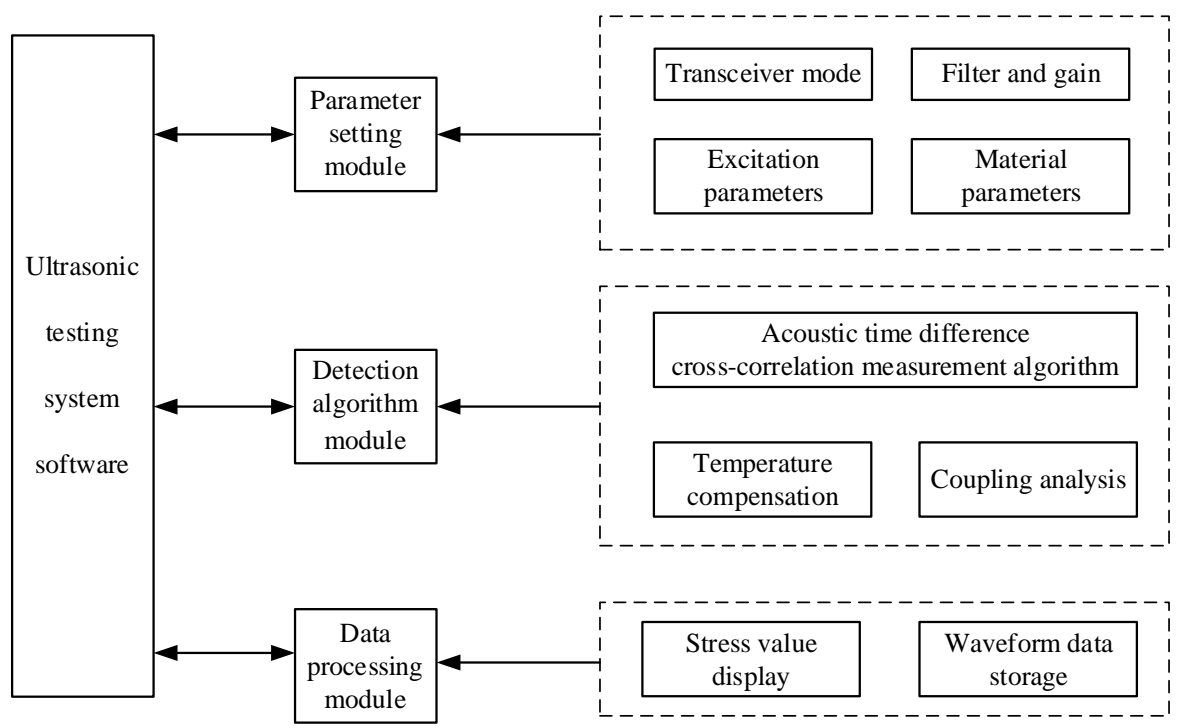

Figure 15. Ultrasonic testing system software structure diagram.

\section{Experimental Verification}

\subsection{Attenuation Coefficient of the RDX/Estane RVE Model}

The explosive powder was compressed into a block, and the RDX mass fraction was $60 \%$ and $40 \%$. The ultrasonic nondestructive testing system was used to detect the PBX samples, adopting the self-sending and self-receiving mode.

The reflectivity of a single ultrasonic transducer with glycerin as the coupling agent, was used to reflect the ultrasonic attenuation coefficient of the sample. The experimental device is shown in Figure 16, which used 1, 2.25, 3, 5, 6, 8, and $10 \mathrm{MHz}$ ultrasonic probes to 
measure the attenuation coefficient. Figure 17 shows the ultrasonic attenuation coefficients of the RDX/Estane PBX with particle volume fractions of $40 \%$ and $60 \%$. Identically, $\mathrm{R}^{2}$ represents the correlation coefficient between the approximating functions and measurement points, and the numbers in brackets beside the data points are the measurement uncertainty (expressed as a composite of the standard deviation of the data and the resolution of the instrument).

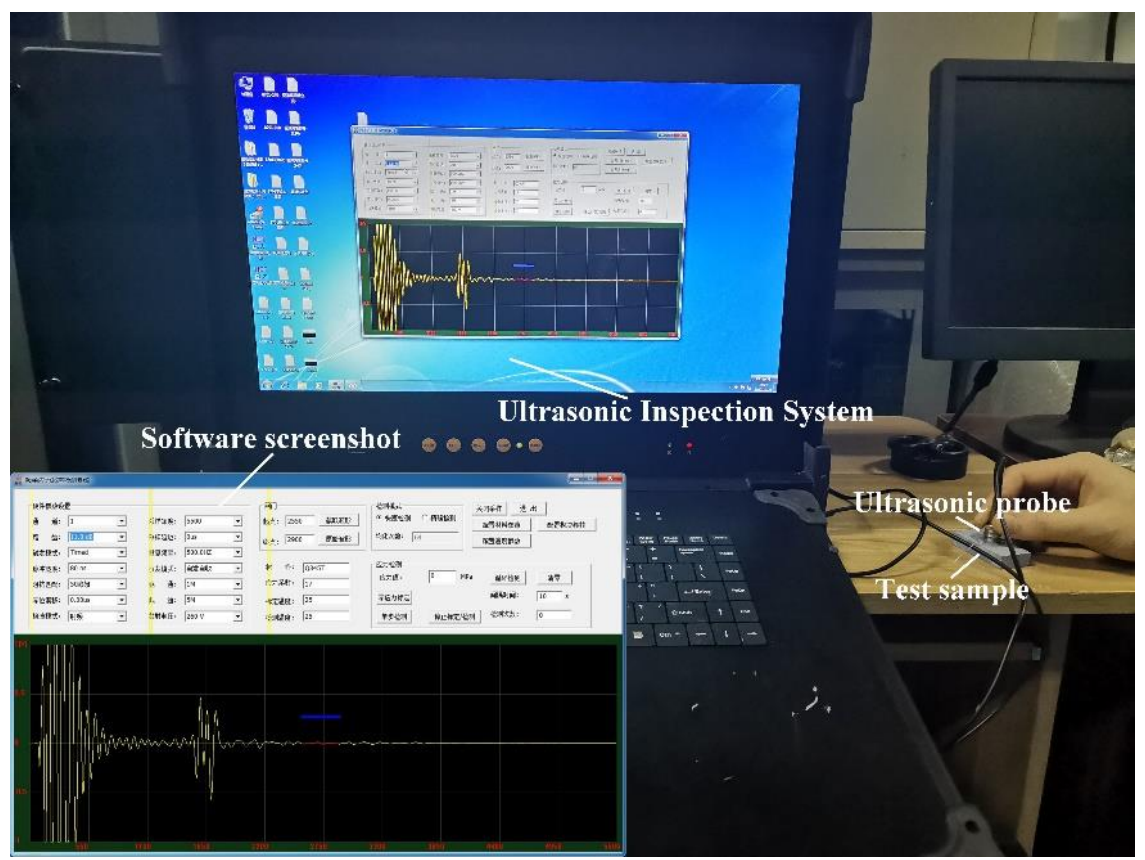

Figure 16. Ultrasonic attenuation coefficient measurement experiment.

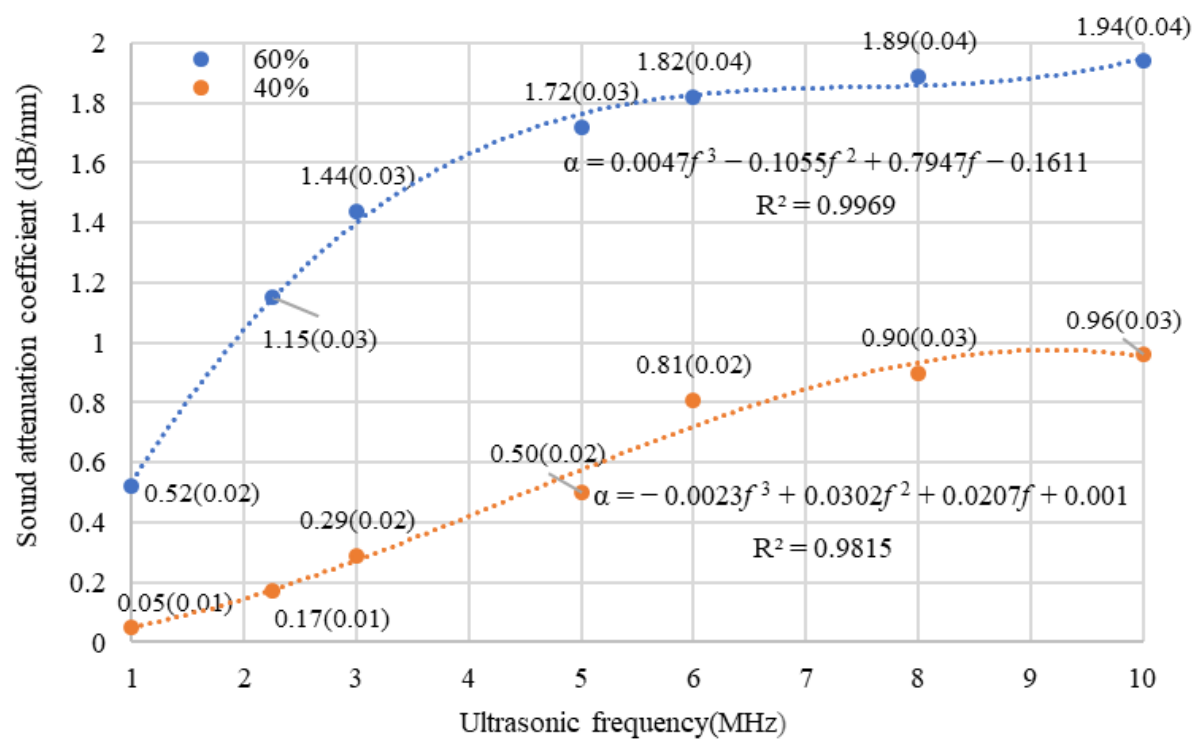

Figure 17. Relationship between the ultrasonic attenuation coefficient and frequency of the RDX/Estane RVE model.

\subsection{PBX Sample Internal Stress Detection}

Using the system described above, stress loading and tests were performed on the PBX to assess the accuracy of the results. Most of the PBXs used in practice are cylindrical. Because the pressed product exhibits better acoustic characteristics, we chose the pressed PBX sample, as shown in Figure 18. The compressed aluminum-containing explosive is made 
by pressing powder with a 60-t high-precision press at China Weapon 204 Research Institute. Its main component is RDX, diameter is $50 \mathrm{~mm}$, height is the elastic modulus is $534 \mathrm{MPa}$, and tensile strength is $9 \mathrm{MPa}$. Furthermore, as ultrasonic waves are severely attenuated during propagation in the PBX, we can only receive reflected low-frequency ultrasonic waves within a certain thickness range. Therefore, we chose a $1 \mathrm{MHz}$ low-frequency ultrasonic probe.

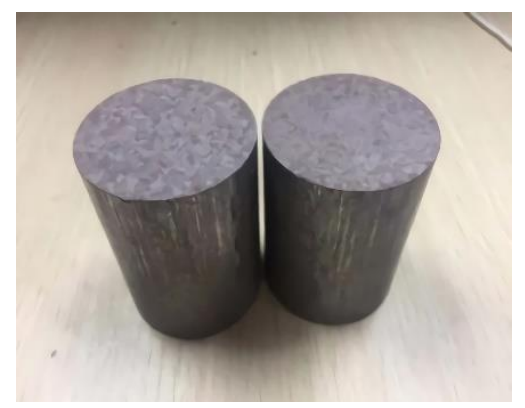

Figure 18. Press-formed PBX sample.

A special fixture was designed to fix the PBX sample and the ultrasonic probe on the worktable of the WDW-200E electronic universal testing machine, as shown in Figure 19. The loading compressive stress maintained the stress gradient at $0.5 \mathrm{MPa}$, and the maximum applied stress was $3 \mathrm{MPa}$. Three sets of compression experiments were carried out, and their respective stress-sound time difference curves were drawn; they exhibited a linear relationship that is in line with the theoretical situation.

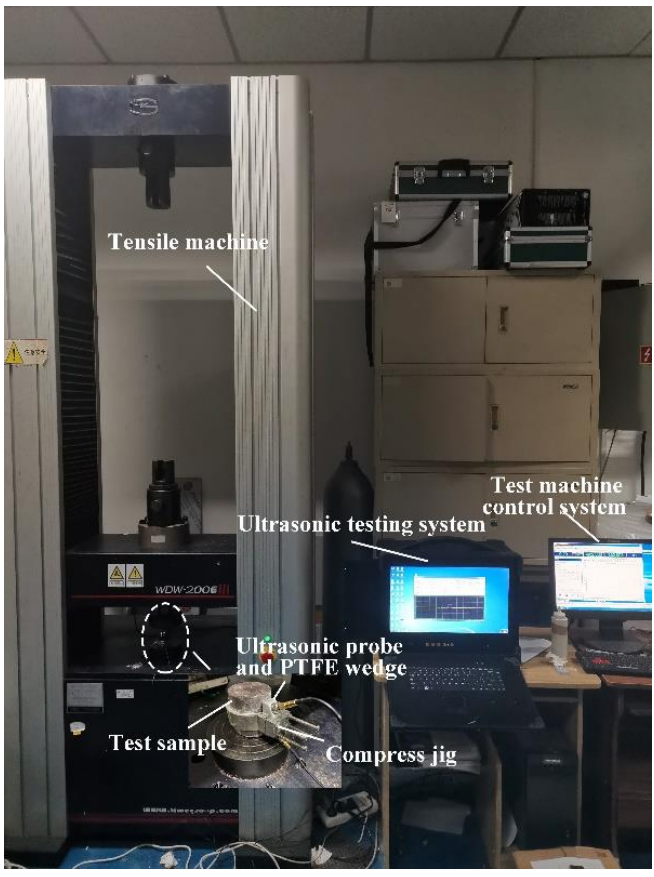

Figure 19. Stress measurement experiment.

As shown in Figure 20, the stress coefficient obtained by multiple experiments is 0.1188 . Identically, $R^{2}$ represents the correlation coefficient between the approximating functions and measurement points, and the numbers in brackets beside the data points are the measurement uncertainty (expressed as a composite of the standard deviation of the data and the resolution of the instrument). To verify the feasibility of ultrasonic nondestructive testing for the internal stress of the PBX material, the measured stress coefficient needs to be calibrated. The specific compressive stress of the PBX sample preset by the tension press was measured, and compared with the actual values. The difference is shown in Table 5. 


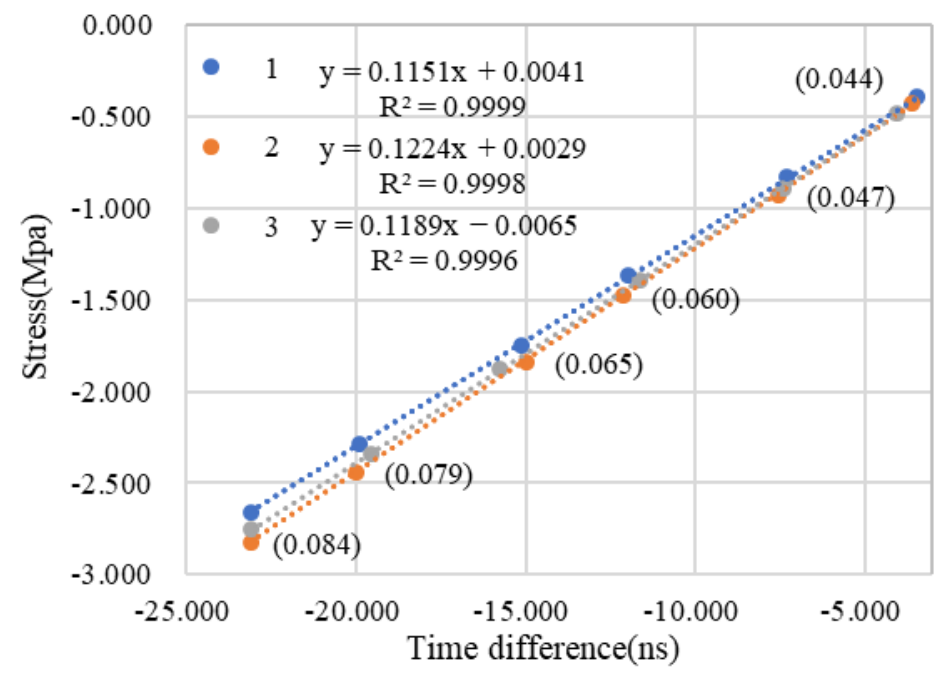

Figure 20. PBX pressure-sound time difference curve.

Table 5. Pressing PBX sample deviation of measurements.

\begin{tabular}{cccccc}
\hline Number & $\begin{array}{c}\text { Applying Load } \\
\text { (MPa) }\end{array}$ & $\begin{array}{c}\text { Measuring Sound Time Difference } \\
\text { (ns) }\end{array}$ & $\begin{array}{c}\text { Measuring Stress } \\
\text { (MPa) }\end{array}$ & $\begin{array}{c}\text { Deviation } \\
\text { (MPa) }\end{array}$ & $\begin{array}{c}\text { Relative } \\
\text { Error }\end{array}$ \\
\hline 1 & -0.5 & 3.69 & -0.438 & 0.062 \\
2 & 7.42 & -0.881 & 0.119 & $12.4 \%$ \\
2 & -1 & 11.91 & -1.415 & 0.085 & $5.67 \%$ \\
3 & -1.5 & 15.29 & -1.816 & 0.184 & $9.20 \%$ \\
4 & -2 & 19.81 & -2.353 & 0.147 & $5.88 \%$ \\
5 & -2.5 & -2.744 & 0.256 & $8.53 \%$ \\
6 & -3 & 23.10 & & & \\
\hline
\end{tabular}

From Table 5, we can see that the difference between the compressive stress value measured by the ultrasonic testing system and that loaded by the tension press is within $15 \%$. This indicates that within a certain tolerance range, the designed ultrasonic testing system is feasible for PBX sample stress testing.

\section{Conclusions}

In this study, a PBX RVE model is established to predict its effective elastic modulus, and an ultrasonic testing system is designed for PBX composite materials. Combined with the experimental results, the following conclusions are drawn:

(1) The effective elastic modulus calculation of the PBX materials can be predicted by parameters such as the explosive particle volume fraction, shape and gradation, and porosity. The RVE model established by Digimat/FE, combined with the load and constraint settings of ABAQUS, can simulate the mechanism of ultrasonic propagation and attenuation inside the material.

(2) The attenuation coefficients of PBX with pure binder and different explosive particle volume fractions were studied. The numerical simulation verified that scattering is the main influencing factor of ultrasonic attenuation, and the attenuation coefficient is positively correlated with the particle volume fraction and ultrasonic detection frequency.

(3) The positive correlation between the ultrasonic attenuation coefficient, the particle volume fraction, and the ultrasonic frequency was verified. The function expression of the ultrasonic attenuation coefficient and the detection frequency was also fitted.

(4) The deviation between the detected stress value and the actual loaded stress value inside the PBX composite material was determined to be within $15 \%$. This indicates that within a certain tolerance range, the designed system is suitable for PBX sample stress testing. 
Author Contributions: Q.P.: conceptualization, resources, supervision, and funding acquisition. S.L.: methodology, validation, and writing-original draft. Y.L.: validation, methodology, and visualization. X.X.: investigation, validation, and formal analysis. M.C.: formal analysis, visualization, and writing-review and editing. Y.Z.: investigation, formal analysis, and writing-review and editing. All authors have read and agreed to the published version of the manuscript.

Funding: This research was funded by the National Natural Science Foundation of China (Grant No. 51975050) and the National Defense Science and Industry Bureau of China (Grant No. JSZL2018602C001).

Informed Consent Statement: Informed consent was obtained from all subjects involved in the study.

Data Availability Statement: The data presented in this study are available on request from the corresponding author. The data are not publicly available due to privacy and secret.

Acknowledgments: We would like to thank Editage (www.editage.cn) for English language editing.

Conflicts of Interest: The authors declare no conflict of interest.

\section{References}

1. Rossini, N.S.; Dassisti, M.; Benyouni, K.Y.; Olabi, A.G. Methods of Measuring Residual Stresses in Components. Mater. Des. 2012, 35, 572-588. [CrossRef]

2. Liu, S.Z.; Rao, N.X.; Zhang, C.; Jin, L. Design of Electromagnetic Ultrasonic Nondestructive Testing System Based on LabVIEW. Trans. China Electrotech. Soc. 2018, 33, 2274-2281.

3. Zhou, H.Q.; Pei, C.X.; Liu, W.W.; Yi, D.C.; Yang, Z.F. Study on Detection Method of Internal Stress in PBX Simulated Material by Laser Ultrasonic Skimming Surface Longitudinal Wave. Chin. J. Energetic Mater. 2018, 26, 786-790.

4. Yang, Z.F.; Tian, Y.; Zhou, H.Q.; Zhang, W.B. Detection of Micro-damages in TATB-based Polymer Bonded Explosive by Nonlinear Ultrasonic Technique. Chin. J. Energetic Mater. 2017, 25, 970-975.

5. Wang, J.C.; Luo, J.R. Predicting the Effective Elastic Modulus of PBX Based on Voronoi Meso-scale Numerical Model. Chin. J. Energetic Mater. 2017, 25, 546-551.

6. Dai, K.D.; Liu, Y.L.; Chen, P.W.; Tian, Y. Finite Element Simulation on Effective Elastic Modulus of PBX Explosives. Trans. Beijing Inst. Technol. 2012, 32, 1154-1158.

7. Zhou, H.P.; Li, J.M.; Li, L.; Wei, X.W.; Zhang, W.B.; Wen, M.P. Testing and Relieving of Residual Stress for Polymer Bonded Explosive Based on TATB. Chin. J. Energetic Mater. 2008, 16, 37-40.

8. Mo, Y.F.; Zhang, X.; Lin, L.; Tian, H.T.; Guang, G.P.; Li, X.M. Investigations on CFRP Porosity by Using Ultrasonic Testing Based on Random Pores Model. J. Mech. Eng. 2010, 46, 22-26.

9. Zhou, X.J.; You, H.W.; Cheng, Y.D. Ultrasonic Attenuation Model of void-contained Carbon- fiber reinforced plastics. Acta Mater. Compos. Sin. 1997, 14, 99-106.

10. Zhou, X.J.; Mo, J.Q.; You, H.W. Ultrasonic Attenuation Testing Method for NDE of Void Content Based on Theoretical Model and Experiment Calibration. Acta Mater. Compos. Sin. 1997, 14, 108-115.

11. Liu, J.Z. Research and Implementation of CFRP Porosity Ultrasonic Nondestructive Testing. Ph.D. Dissertation, Machinery and Energy Engineering College Zhejiang University, Zhejiang, China, 2005.

12. Yu, Y.L.; Ye, J.R.; Liu, K.; Zhang, B.M. A Mesoscale Ultrasonic Attenuation Finite Element Model of Void-containing Composites. Acta Mater. Compos. Sin. 2014, 31, 171-178.

13. Han-song, H.; Ramesh, T. Effects of Void Geometry on Elastic Properties of Unidirectional Fiber Reinforced Composites. Compos. Sci. Technol. 2005, 65, 1964-1981.

14. Wang, D.Y.; Wen, W.D.; Cui, H.T. Three-dimensional Progressive Damage Analysis of Composite Laminates Containing a Hole Subjected to Tensile Loading. Acta Mech. Sin. 2005, 7, 118-125.

15. Xie, Q.; Li, Y.Z.; Li, B.; Li, X. The Strength Prediction of Composite Laminates Based on a Quasi-3D Finite Element Models. Sci. Technol. Eng. 2012, 12, 3160-3165.

16. Pan, Q.X.; Shao, C.; Xiao, D.G.; Pan, R.P.; Liu, X.H.; Song, W. Robotic Ultrasonic Measurement of Residual Stress in Complex Curved Surface Components. Appl. Bionics Biomech. 2019, 2019, 2797896. [CrossRef]

17. Yashar, J.; Mehdi, A.K.; Mehdi, A.N. Residual Stress Evaluation In Dissimilar Welded Joints Using Finite Element Simulation And The LCR Ultrasonic Wave. Int. J. Innova. Technol. Manag. 2013, 4, 170-174.

18. Pan, Q.X.; Li, Y.; Xu, C.G.; Xiao, D.G.; Yang, X.C.; Wu, Y. Residual Stress Measurement for Welded Structure by Ultrasonic Wave. Electro. Mech. Eng. 2012, 28, 8-12.

19. Xu, C.G.; Song, W.T.; Pan, Q.X.; Li, X.; Jin, X.; Liu, H.Y. Residual Stress Nondestructive Testing Method Using Ultrasonic. Nondestr. Test. 2014, 36, 25-31.

20. Xu, C.G.; Li, H.X.; Wang, J.F.; Pan, Q.X.; Xiao, D.G. Ultrasonic Shear and Longitudinal Wave Testing Method of Residual Stress. Acta Acoust. 2017, 42, 195-204. 
21. Yang, Y.M.; Ju, Y.; Liu, H.B.; Wang, H.J. Influence of Porous Structure Properties on Mechanical Performances of Rock. Chin. J. Rock Mech. Eng. 2009, 28, 2031-2038.

22. Kang, G.; Chen, P.W.; Guo, X.; Ma, G.W.; Ning, Y.J. Simulations of Meso-scale Deformation and Damage of Polymer Bonded Explosives by The Numerical Manifold Method. Eng. Anal. Bound. Elem. 2018, 96, 123-137. [CrossRef]

23. Liu, Y.L. Micro-mechanical numerical simulation of viscoelastic behavior of PBX explosives. In Proceedings of the 20th Annual Academic Conference of the Beijing Society of Mechanics, Beijing, China, 12 January 2014.

24. Zhang, Y.G.; Lou, J.F.; Hong, T. Modification of Viscose-Statistical Crack Mechanics for PBXs. Chin. J. High. Press. Phys. 2015, 29, 9-14.

25. Gao, J.; Huang, Z.X. Parameter Sensitivity Analysis for Viscoelastic Damage Constitutive Model of PBX Materials. Eng. Mech. 2015, 32, 201-206.

26. Djerir, W.; Ourak, M.; Boutkedjirt, T. Characterization of the Critically Refracted Longitudinal (LCR) Waves and Their Use in Defect Detection. Res. Nondestr. Eval. 2014, 25, 203-217. [CrossRef] 